\title{
Análise Crítica da Carta Brasileira de Prevenção Integrada na Área da Saúde na Perspectiva da Educação Física Através do Enfoque Radical de Promoção da Saúde
}

Critical Analysis of the Brazilian Charter of Integrated Prevention in the Field of Health in the Perspective of Physical Education Through the Radical Focus of Health

\section{Promotion}

\section{Fabio F. B. de Carvalho}

Especialista em Saúde Pública - Escola Nacional de Saúde Pública Sergio Arouca/Fundação Oswaldo Cruz.

Endereço: Av. 28 de Setembro, 397 apto 304 fundos, Vila Isabel, CEP 20551-030, Rio de Janeiro, RJ, Brasil.

E-mail: fabiofbcarvalhoœgmail.com

\section{Resumo}

O presente trabalho pretende contribuir para que a área profissional e o campo do saber referente à Educação Física apreendam a saúde de forma ampliada, superando o enfoque biologicista hegemônico. Possui caráter qualitativo e baseou-se em pesquisa de fontes bibliográficas para a confecção de monografia de conclusão do curso de especialização em Saúde Pública, na Escola Nacional de Saúde Pública Sergio Arouca da Fundação Oswaldo Cruz. Analisou-se criticamente a Carta Brasileira de Prevenção Integrada na área da Saúde na perspectiva da Educação Física - publicação do Conselho Federal de Educação Física (CONFEF), em 2006, a partir do enfoque radical da Promoção da Saúde. Esse enfoque pode ser sintetizado na proposta de articulação entre saúde e condições de vida, ressaltando as inter-relações de equidade social, através do aumento da capacidade da participação popular, intersetorialidade, fomento de políticas públicas saudáveis, criação de ambientes favoráveis à saúde e reorientação do sistema de saúde, baseando-se numa visão ampliada do processo saúde-doença e reconhecendo a multideterminação desse processo (OMS, 1986; Buss, 2000, 2001, 2007; Pedrosa, 2004). Concluiu-se que o documento analisado biologiza e medicaliza a Educação Física em sua relação com a sociedade. Recomenda-se que o mesmo seja revisto a partir da perspectiva crítica ligada à área da Educação Física, a qual relaciona a atividade física e a saúde com questões sociais mais 
amplas que impedem que os sujeitos tomem decisões mais saudáveis, tais como os condicionantes econômicos, culturais, étnicos e políticos.

Palavras-chave: Promoção da saúde; Educação física; Saúde; Atividade física.

\section{Abstract}

This work intends to provide a contribution so that the professional area and the field of knowledge referring to Physical Education view health in a broader way, overcoming the biological focus which usually occurs. It has a qualitative character and was based on research into bibliographic sources in order to develop a monograph upon the completion of the Public Health specialization course at the National School of Public Health Sergio Arouca, of the Oswaldo Cruz Foundation. The Brazilian Charter of Integrated Prevention in the Field of Health in the Perspective of Physical Education, a document written by the Federal Council of Physical Education (CONFEF) in 2006, was critically analyzed based on the radical focus of Health Promotion. This focus can be summarized as an articulation between health and life conditions, emphasizing social equity interrelations, through the increase in the capacity for popular participation, intersectoriality, promotion of healthy public policies, creation of environments that are favorable to health, and through a reorientation of the health system, based on a broader view of the health-disease process and on the recognition of the multi-determination of this process (OMS, 1986; Buss, 2000, 2001, 2007; Pedrosa, 2004). We concluded that the document that we analyzed assumes a biological and medical view concerning the relation between Physical Education and society. We recommend that this document is revised based on the critical perspective connected with the Physical Education field, which relates physical activities and health to broader social issues which prevent subjects from making healthier decisions, such as economic, cultural, ethnic and political conditioning factors.

Keywords: Health Promotion; Physical Education; Health; Physical Activity.

\section{Introdução}

O presente trabalho justifica-se como uma tentativa de ampliar a interlocução entre os referenciais da Promoção da Saúde com os da Educação Física mediante a análise crítica da Carta Brasileira de Prevenção Integrada na área da Saúde na perspectiva da Educação Física - documento redigido pelo Conselho Federal de Educação Física (CONFEF). Torna-se relevante ao contribuir para que essa área profissional e campo do saber apreendam a saúde de forma ampliada, superando a enfoque biologicista hegemônico, conforme apontaram Deslandes (2004), Bagrichevsky e Estevão (2005), Carvalho (2005), Darido e Sanches Neto (2005).

No século XIX, Virchow, Villermée, Chadwick, entre outros, já apontavam as relações entre as condições de vida e saúde (Rosen, 1994). Em meados dos anos 1980, como reação à acentuada medicalização da saúde na sociedade e no interior do sistema de saúde ou, ainda, devido ao custo crescente da assistência médica sem que ocorressem resultados igualmente significativos as ideias sobre promoção da saúde foram introduzidas no Brasil em consonância com o movimento que ocorria em âmbito mundial. Assim, trata-se de um conceito direcionado aos determinantes gerais da saúde, entendendo-a como um produto histórico e social (Buss, 2000, 2003; Czeresnia, 2003; Carvalho, 2008). Segundo a Carta de Ottawa, documento que deu início ao movimento da Promoção da Saúde, caracteriza-o por ser um "processo de capacitação da comunidade para atuarna melhoria de sua qualidade de vida e saúde, incluindo uma maior participação no controle deste processo" (Organização Mundial da Saúde - OMS, 1986). Segundo Reis e colaboradores (2002), essa carta ampliou a concepção de promoção da saúde naquele determinado momento histórico, pois incorporou a importância e o impacto dos aspectos sociais, econômicos, políticos e culturais nos determinantes da saúde. Assim, as ações de promoção da saúde objetivam fazer com que as condições de vida sejam cada vez mais favoráveis à vida saudável ou com qualidade (OMS, 1986).

Em 2006, o Conselho Federal de Educação Física (CONFEF) publicou um documento denominado Carta Brasileira de Prevenção Integrada na Área da Saúde na perspectiva da Educação Física, objeto de análise deste trabalho, que em sua apresentação, reconhece a importância da prevenção, da promoção da saúde e do papel essencial do Profissional de Educação Física na composição de políticas públicas de saúde. 


\section{Métodos}

O presente trabalho possui caráter qualitativo e baseou-se em pesquisa de fontes bibliográficas para a confecção de monografia de conclusão do curso de especialização em Saúde Pública, na Escola Nacional de Saúde Pública Sergio Arouca da Fundação Oswaldo Cruz (ENSP/FIOCRUZ). A partir da leitura da Carta Brasileira de Prevenção Integrada na Área da Saúde na perspectiva da Educação Física, documento redigido pelo CONFEF em 2006, buscou-se analisá-la a partir do enfoque radical da Promoção da Saúde, como denominado por Buss (2007).

\section{Resultados e Discussão}

\section{Promoção da Saúde: algumas implicações}

A Promoção da Saúde propõe uma articulação de saberes técnicos e populares, a mobilização de recursos institucionais e comunitários, públicos e privados, ou seja, entre saúde e condições de vida, ressaltando as inter-relações de equidade social, através do aumento da capacidade da participação popular, intersetorialidade, fomento de políticas públicas saudáveis, criação de ambientes favoráveis à saúde e reorientação do sistema de saúde, baseando-se numa visão ampliada do processo saúde-doença e reconhecendo a multideterminação desse processo (OMS, 1986; Buss, 2000, 2001; Pedrosa, 2004). Assim, o foco está na sociedade, nas relações que interagem com a saúde e no ambiente, compreendido em seus aspectos físico, social, político, econômico e cultural e na vulnerabilidade do coletivo, e que irão requerer intervenções articuladas intersetorialmente e participação social com o objetivo de conceber a saúde como direito. Portanto, a saúde envolve os demais setores da sociedade, além do setor médico e dos demais profissionais da saúde (Palma e col., 2003; Czeresnia, 2003; Marcondes, 2004; Pedrosa, 2004).

Assim,

a promoção da saúde deve ser compreendida como uma agenda integrada e multidisciplinar cujo objetivo primordial é fomentar mudanças em três níveis: assistência à saúde, gestão local de políticas públicas e proteção e desenvolvimento sociais para todos (Carvalho, 20o8, p. 4).
Para Campos e colaboradores (2004), falar da promoção da saúde no Brasil é indissociável da reflexão e enfrentamento das iniquidades sócio-sanitárias que envolvem não só o setor saúde. Assim, os autores refletem sobre como ela pode auxiliar na "geração de outros modos de atenção e de gestão da saúde, de criação do viver e de outras realidades" (Campos e col., 2004, p. 746).

Czeresnia (2003); Campos e colaboradores (2004) afirmam ser a autonomia dos sujeitos e coletividades através da capacitação para o processo do cuidado à saúde um dos principais pilares da promoção da saúde. Zioni e Westphal (2007) concordam com tal afirmativa ao entenderem que a promoção da saúde ao se propor a compreender as condições de saúde, como elas são produzidas e advogar a participação popular no processo de tomada de decisões, deve tornar os indivíduos e coletividades capazes de agirem com protagonismo nas decisões que impliquem em sua saúde. Assim, segundo essas autoras, deve ocorrer o "reconhecimento da autonomia individual e coletiva como elementos criadores de novas alternativas e, portanto, transformadores da dinâmica social e da sociedade" (p. 28). Peres e colaboradores (2005) relatam que a efetividade de ações de promoção da saúde, ou seja, que objetivam a melhoria da qualidade de vida e, consequentemente, da saúde implicam um profundo envolvimento dos atores e da população beneficiária. Marcondes (2004), por sua vez, afirma que a diferenciação entre a promoção da saúde ser instrumento do neoliberalismo, ou seja, advogar uma perspectiva de retração de investimentos públicos em políticas sociais, ou de igualdade social, de fato, será a sua vinculação à participação social.

Para Andrade e colaboradores (2004) podemos, hoje, perceber melhor o nosso estado de saúde, e assim nos tornarmos mais autônomos e mais responsáveis por nossa qualidade de vida. Isso devido à melhoria geral das condições alimentares, de moradia, de saneamento básico, de educação e de informação. Contudo, cremos não serem suficientes apenas tais melhorias, devido à grande parcela das populações brasileira e mundial não ter, ainda, acesso a elas.

Marcondes (2004) aponta três questões referenciais para se debater a promoção da saúde, quais sejam: as limitações do modelo biomédico, a ampliação do conceito de saúde e a diferença entre prevenção e promoção. 
O modelo biomédico baseou-se na doutrina da etiologia específica que consistia na crença de que, para cada enfermidade, haveria um único agente específico, assim o contexto de vida dos sujeitos foi deixado de lado na consideração do processo saúde-doença (Farinatti e Ferreira, 2006). Barros (2002) considera o fenômeno da medicalização como consequência e estímulo à hegemonia do modelo biomédico que encontra ambiente propício na sociedade de consumo, sob o império da lógica de mercado. Esse autor define medicalização como "a crescente e elevada dependência dos indivíduos e da sociedade para com a oferta de serviços e bens de ordem médico-assistencial e seu consumo cada vez mais intensivo" (p. 7). Farinatti e Ferreira, por sua vez, definem-na como "invasão de uma quantidade importante de setores da vida cotidiana pelas determinações da medicina" (p. 135), o que gera um entendimento de que a saúde é área exclusiva da medicina.

Barros (2002) lembra-nos que não se trata de culpabilizar de forma simples e linear o modelo biomédico sem considerar como se deu essa hegemonia ao longo da história. Destaca-se que não é objetivo deste estudo aprofundar-se nessa discussão a respeito desse modelo e, sim, apenas situá-lo no debate que a promoção da saúde busca fomentar. Posteriormente, percebeu-se que o paradigma biomédico era insuficiente para responder de forma satisfatória os componentes subjetivos presentes no processo saúde-doença. Assim, para Barros (2002), há profissionais - o autor fala especificamente do profissional médico, mas acreditamos poder ampliar para qualquer profissional de saúde - que compreendem a importância de visualizar o sujeito como um todo relacionado ao seu contexto socioeconômico, contudo terminam por regressar ao reducionismo, pois esse foi o modelo em que foi pautada sua formação.

Marcondes (2004), em consonância com este estudo, defende que a compreensão da saúde relaciona-se às condições de vida que são geradas pelas relações sociais - já que estas permeiam o acesso a alimentação, educação, trabalho, renda, lazer, paz e ambientes saudáveis, entre outros aspectos fundamentais para a saúde e a qualidade de vida; assim, são importantes elementos do processo saúde-doença. Continua, afirmando que a ampliação do conceito de saúde partiu do reconhecimento da limitação do foco exclusivo no risco individual para responder aos processos de saúde-doença baseados em modelos biológicos e de tecnologias médicas (modelo biomédico).
Esse autor alerta ainda sobre a interdependência entre saúde e desenvolvimento e afirma que o crescimento econômico deve beneficiar as condições de vida da população como um todo para que os setores sociais e sanitários sejam efetivos ao se mobilizar para buscar gradações cada vez maiores de saúde individual e coletiva, assim como propõe o emprego da promoção da saúde para a adequação de políticas, programas e serviços, como forma de enfrentar tanto a medicalização da vida social como a individualização da saúde pública. A diferença entre promoção e prevenção será abordada em item posterior.

\section{Promoção da Saúde Crítica ou Conservadora?}

O conceito de promoção da saúde tem sido interpretado de diferentes formas ao longo de sua construção, ou seja, numa perspectiva mais conservadora e noutra crítica, como denominaram Farinatti e Ferreira (2006). Na primeira, o foco seria a redução do peso econômico dos serviços de saúde ao imputar ao indivíduo a responsabilidade pela sua saúde, independente do contexto de vida deste. E, na segunda, o foco seria a transformação social através da capacitação dos cidadãos. Czeresnia (2003) utiliza os termos perspectivas conservadoras e progressistas, respectivamente.

Buss (2001), Ferreira e Buss (2002), Palma e colaboradores (2003) corroboram ao afirmarem existir dois principais grupos onde podem ser reunidas as conceituações de promoção da saúde. Em um deles, estariam fora do âmbito da promoção da saúde todos os fatores que estivessem fora do controle dos indivíduos, ou seja, as atividades são dirigidas a mudanças comportamentais dos indivíduos. No entanto, Buss (2001) se alinha com o que denomina "conceito moderno de promoção da saúde" (p. 279) ou, como denominado mais recentemente. Enfoque radical de Promoção da Saúde (Buss, 2007), no qual a saúde é produto de um amplo espectro de fatores relacionados à qualidade de vida; logo, as atividades estariam mais voltadas ao coletivo de indivíduos e ao ambiente, entendido em seu contexto físico, social, político e econômico. Autores como Peres e colaboradores (2005), Stotz e Araújo (2004) também apontam a existência de duas concepções distintas de promoção da saúde, estes últimos ainda afirmam que ambas foram incorporadas pela OMS.

Não se trata de polarizar ou rivalizar ambas as concepções de promoção da saúde, já que necessariamente haverá mudanças individuais para que indivíduos e 
coletivos alcancem gradações cada vez maiores de saúde, contudo, tais mudanças comportamentais viriam associadas a mudanças nos contextos socioeconômico e cultural. Logo, o que está em voga é o enfoque e a predominância de qual concepção de promoção da saúde será utilizada.

\section{Promoção X Prevenção}

Há ainda, em muitos casos, uma indefinição conceitual que gera uma confusão entre práticas ditas de promoção da saúde e de prevenção. Czresnia (2003) explicita definições que nos auxiliam a distingui-las. Para ela a prevenção estrutura-se mediante a divulgação de informação científica e de recomendações normativas de mudanças de hábitos. Já a promoção extrapola a aplicação técnica e normativa, pois objetiva o fortalecimento das capacidades individual e coletiva para a compreensão e o enfrentamento dos condicionantes da saúde, e complementa: "essa concepção diz respeito ao fortalecimento da saúde pormeio da construção da capacidade de escolha" (2003, p. 48) e "isso não é da esfera do conhecimento verdadeiro, mas do valor" (2003, p. 49).

0 quadro I traz algumas diferenças entre as abordagens da promoção e da prevenção.

\section{Quadro I}

\begin{tabular}{l|l} 
Promoção & Prevenção \\
\hline Determinantes da saúde, criar alternativas saudáveis & Reduzir os fatores de risco e enfermidades \\
\hline População em geral e as condições relacionadas à saúde & Indivíduos com possibilidade de adoecer, àqueles com elevado risco \\
\hline Conteúdos sociopolíticos, ecológicos e socioculturais & Ênfase aos fatores de risco, práticas clínicas, preventivas e reabilitação \\
\hline
\end{tabular}

Adaptado de Palma colaboradores (2003).

Contudo, não há porque prescindir dessas formas de atenção à saúde, pois para Carvalho (2005) não há limites rígidos entre elas. A autora também concorda que a principal diferença entre elas é a ênfase com que são utilizadas e sugere que a clínica incorpore o social e o subjetivo, assim como a Saúde Pública/Promoção da Saúde atue com o auxílio da clínica.

\section{Atualidade do Debate sobre a Promoção da Saúde}

Através do discurso de posse do atual Ministro da Saúde, José Gomes Temporão, percebe-se o quão atual é o debate que o ideário da promoção da saúde busca, pois este assumiu que "vivemos um intenso processo de medicalização perdendo-se a perspectiva de que, o que mais produz saúde [...] está fora da governabilidade setorial" (Temporão, 2007). Ele referia-se a renda, emprego, habitação, saneamento, lazer, cultura, educação entre outros, portanto [...] "a saúde é, antes que biológica, uma produção social” (Temporão, 2007).

Ainda afirmou pretender assumir "uma visão integrada interinstitucional, múltipla e interativa que aproxime os espaços da saúde, educação, esportes, cultura, saneamento, segurança, habitação com as políticas de inclusão social". Há também nesse discurso o evidenciamento de que se torna necessário ampliar o debate proferido pela Saúde Coletiva/Promoção da Saúde na Educação Física, já que esta lida diretamente com educação, lazer, cultura, esportes, entre outros.

\section{Carta Brasileira de Prevenção Integrada na Área da Saúde na Perspectiva da Educação Física}

Em 2006, o CONFEF redigiu este documento no qual há o privilegiamento da prevenção, como buscaremos evidenciar. A Carta, em sua introdução, defende a atividade física pelas múltiplas potencialidades que possui, afirmando que o esporte, a ginástica, a dança, as lutas, a musculação, apresentam-se como "práticas economicamente viáveis para promoção da saúde e da inclusão social, revelando-se ícones da prevenção integrada e holística" (p. 4). Leva-nos a reflexão do que se entende, neste documento, por Promoção da Saúde, já que quase sempre traz os campos da prevenção e promoção da Saúde como sinônimos.

Para este documento a

atividade física sistematizada e o Esporte, quando conduzidos e orientados de forma adequada, formativa, educativa, qualificada e ética, ou seja, por Profissional de Educação Física habilitado, por representarem atividades lúdicas e livres que, 
como atividades socializantes por si só, atraem as pessoas, são considerados por todos os diferentes segmentos de profissionais da área da Saúde como excelentes meios minimizadores e facilitadores na busca de saídas e soluções para os problemas que vêm recrudescendo de forma progressiva na sociedade atual (p. 4).

Transparece certo otimismo ingênuo ao acreditar que o profissional de educação física, simplesmente por ser habilitado, conseguirá orientar da forma citada toda e qualquer atividade física e/ou desportiva. Não leva em conta que esse profissional está inserido numa sociedade que pode dar mais ou menos valor para a atividade, sua atuação profissional, o que acarretará, entre outros, mais ou menos condições de trabalho para esse profissional desde sua formação inicial até a inserção no mercado de trabalho. Além disso, permite o entendimento da atividade física e/ou desportiva como uma espécie de 'salvadoras da pátria', imputando a elas um poder que acreditamos estar mais em consenso no senso comum do que de fato em evidências.

Érazoável aceitar que a prática regular de atividade física traz benefícios para a saúde (Ferreira e Najar, 2005; Palma e col., 2003; Bagrichevsky e Estevão, 2005), esta entendida aqui em seu conceito ampliado, não se restringindo apenas ao seu componente biológico. Portanto, entendemos a saúde como produção social, a qual está intimamente ligada à qualidade de vida, o que a torna um direito inalienável de todo e qualquer sujeito.

O documento aproxima-se, em determinados momentos, do referencial da Promoção da Saúde defendido por este texto e do conceito ampliado de saúde, por exemplo, ao afirmar sobre o direito à saúde que "as ações de prevenção e promoção da Saúde são estratégias positivas de intervenção para a busca contínua de avanços políticos, sociais, culturais, ambientais $e$ educacionais, sempre no sentido do desenvolvimento humano" (p. 13). E, ainda, ao entender a saúde como direito humano fundamental e essencial, como fator de investimento social e econômico, ou que "a busca da saúde pressupõe o exercício da cidadania, significando assumir a responsabilidade com a qualidade de vida" (p. 5). E ainda que "os campos da Prevenção e da Promoção têm se deslocado progressivamente para o centro das atenções na área da saúde, da educação, da economia, do trabalho, da justiça social, [...] tanto no setor público como no privado" (p. 5). Contudo, mais uma vez traz prevenção e promoção da saúde de forma quase indissociável, além de defender uma relação da qualidade de vida "com um estilo de vida ativo, de modo individual e participativo na comunidade" (p. 5).

Continua, defendendo que a profissão Educação Física, quando aplicada de forma qualificada, competente, responsável e ética, certamente poderá contribuir significativamente para a melhoria da qualidade de vida da comunidade e fortalecimento dos anseios e dos direitos de cidadania. 0 que não fica claro e já foi motivo de ressalva neste texto é: como caracterizar a forma qualificada, competente, responsável e ética do profissional de Educação Física? Apenas a formação acadêmica e a habilitação profissional poderiam fazê-lo? O documento afirma que, dinamizada competentemente, essa Educação Física proporcionará a ampliação e o reconhecimento da importância de serviços prestados pela categoria dos profissionais da área. Nisso concordamos e esse é um dos objetivos deste trabalho e da discussão que o mesmo pretende fomentar, só que não há como fazer esse debate de forma simplista, imputando ao profissional de educação física ou à atividade física e/ou desportiva um estatuto de solução para "grande parte dos males da modernidade" (p. 6) ou ainda que seria permitido ao indivíduo a partir da adoção de um estilo de vida ativo "condições de viver e conviver livremente com suas necessidades, desejos [...] podendo exercer todos os seus direitos e capacidades de cidadania" (p. 6). É como se estes fossem bons por si só, independente do contexto onde se dá ou daquele que os pratica. Questiona-se: ser ativo proporcionaria condições de viver a todos? Só isso é necessário para a cidadania?

Enfatiza como benefícios da atividade física apenas os que estimulam os vários sistemas orgânicos; mas afirma, por diversas vezes, que ela tem muitos benefícios quanto à inclusão social. Ora, questionase: aspectos biológicos como melhora no sistema cardiopulmonar, manutenção da pressão sanguínea em níveis aceitáveis, entre outros, são suficientes para a problemática social que a sociedade brasileira enfrenta? Ao defenderem a atividade física como "uma arma eficaz para a promoção da saúde" (p. 6), o fazem biologizando a questão, pois afirmam que "o aumento da atividade física tende a reduzir em 1/3 os casos de doenças arteriais e coronarianas, bem como para con- 
trole dos fatores de risco e um poderoso caminho para a melhoria da qualidade de vida"(p. 6). 0 que nos permite inferir que, para esse documento, a qualidade de vida restringir-se-ia a ausência ou atenuação de doenças. É importante ressaltar que o aspecto biológico da Saúde deve também ser privilegiado, contudo, quando apenas este é abordado, acaba por ocorrer a aproximação com o modelo biomédico que já foi, de forma sucinta, objeto de análise no presente texto.

Afirma que a prática regular de atividades físicas possui relevância na formação do ser humano e na conquista da qualidade de vida; porém as classifica como pertencentes ao aspecto economia. Este trabalho sugere a reflexão sobre o entendimento de que a formação do ser humano e a atividade física como importante instrumento para tal, sirvam apenas como poupadores de possíveis gastos já que, por essa ótica, acreditar-se-ia que atividade física poderia prevenir diversas doenças crônicas não transmissíveis (DCNT). O que fica explicitado através do entendimento da Carta de que a atividade física seria um meio 'eficiente e barato' de promoção da saúde.

$\mathrm{O}$ documento acredita que a atividade física faz com que "as pessoas tenham mais energia e disposição para aproveitar o seu envolvimento tanto no mundo do trabalho, como do lazer e social" (p. 6). Mostra um descolamento da realidade de vida de muitos brasileiros, pois é sabido que existem jornadas de trabalho esgotantes (somando-se deslocamento, jornada de trabalho propriamente dita e afazeres domésticos) e, portanto, não haveria tempo ou 'energia' para o engajamento em atividades físicas, ou ainda, há a possibilidade de que a atividade física realizada na atividade laboral seja esgotante e não aprazível ao sujeito que a pratica. Palma e colaboradores (2007) trouxeram essa problemática para acadêmicos e futuros profissionais de Educação Física; a partir daí pode-se inferir que nós, profissionais de Educação Física e de saúde, muitas vezes não conseguimos realizar atividades físicas para ter mais 'energia', como costumeiramente recomendamos, mas mesmo assim acreditamos que outrem tem de praticá-las, independente dos diversos fatores intervenientes.

Cita importantes documentos como a Agenda de Berlim (1999), que defende: “a Educação Física é um direito humano de todas as pessoas, principalmente das crianças” (p. 9); ou a Declaração de São Paulo para a Promoção da Atividade Física no Mundo (1999), que considera que "aproximadamente dois milhões de mortes por ano no Planeta são atribuídas ao sedentarismo e que a inatividade física associada ao fumo e pobreza causa $75 \%$ das mortes de doenças crônicas não transmissíveis" (p. 10). Só que não traz em seu corpo nenhuma proposta para combater a pobreza, o que, a nosso ver, restringe a efetividade da prática de atividade física para evitar tais mortes.

Em determinado momento, esclarece seu posicionamento de tratar prevenção e promoção da saúde de forma quase indissociável, ao defender que elas "devem ser consideradas conjuntamente pelas suas proximidades e raízes comuns no bem-estar de cada indivíduo" (p. 14). Já foram apontadas neste trabalho algumas diferenças entre elas. Diferenças tais que nos fazem optar por enfatizar a promoção da saúde como principal forma de abordar a atividade física, como importante fator para a saúde.

A Carta aponta para a necessidade de compartilhamento entre as pessoas, suas famílias, comunidades, organismos e instituições e do Estado para a prevenção e promoção da saúde e, ainda, que estas serão reforçadas em sociedades mais justas e cooperativas, onde as políticas públicas conseguiriam maior possibilidade de êxito social. Reitera a interdisciplinaridade e intersetorialidade como essenciais para a prevenção e promoção da Saúde.

Cita também a educação para a saúde, pois “possibilita a tomada de consciência, a participação, a consciência crítica do momento histórico vivido e a formação de uma possível rede de suporte social [...]" (p. 18). De forma semelhante ao que ocorre com a Promoção da Saúde e foi evidenciado neste texto, a educação em saúde pode se constituir em práticas impositivas, com o objetivo de prescrever comportamentos "ideais" e que seriam desvinculados da realidade de vida desses sujeitos, ou a instrumentalização da sociedade, com o objetivo de participar de intervenções na realidade concreta de vida de cada sujeito, buscando a qualidade de vida, ou seja, uma educação para a cidadania (Pedrosa, 2006). Parece-nos que a educação para a saúde advogada pelo documento se aproxima do que foi descrito primeiramente.

No texto analisado prevalece um cunho corporativista ao permitir, por exemplo, o entendimento de que apenas pela 'perspectiva da Educação Física' é 
que ocorreria um processo educacional, formal ou não formal, na prática de atividades físicas.

Conclui que os profissionais de Educação Física, a partir do entendimento de que essa área se relaciona com a educação, saúde, cultura, esporte, lazer, “[...] terão na sua atuação por estilos de vida ativos na população do país, que valorizar a educação para a saúde" (p. 22).

Castiel (2003, p. 93) traz importante reflexão sobre uma possível busca por 'estilos de vida ativos' ao afirmar que

uma crítica comum ao conceito 'estilo de vida' é referente a seu emprego em contextos de miséria e aplicado a grupos sociais onde as margens de escolha praticamente inexistem. Muitas pessoas não elegem 'estilos' para levar suas vidas. Não há opções disponíveis. Na verdade, nestas circunstâncias, o que há são estratégias de sobrevivência [grifo do autor].

Mesmo quando não se trata dos contextos de miséria citados, as opções são definidas em maior ou menor parte pelo contexto no qual estamos inseridos, o que é explicado por Buss e Pellegrini Filho (2007), ao afirmarem que os estilos de vida e comportamentos são fortemente influenciados, por exemplo, pela pressão dos pares, propaganda, possibilidade de acesso a alimentos saudáveis, espaços de lazer, entre outros. A Política Nacional de Promoção da Saúde também auxilia nessa reflexão ao afirmar que "os modos de viver não se referem apenas ao exercício da vontade e/ou liberdade individual e comunitária" (Brasil, 2006, p. 11).

\section{Considerações}

O documento biologiza e medicaliza a Educação Física em sua relação com a sociedade ao afirmar, por exemplo, que a "prevenção como fator estratégico no combate às injustiças sociais, à violência, ao analfabetismo, à evasão escolar, à exclusão social" (p. 4); ou ainda quando afirma que "prevenção significa antecipação, leitura prévia e identificação de situações sociais inadequadas que, a curto, médio e longo prazos, possam trazer prejuízos à população" (p. 4). Não fica claro o que se deve prevenir para combater as injustiças sociais e a violência, entre outros, e quais sugestões para o enfrentamento dessas situações sociais inadequadas, a partir de sua leitura prévia e identificação.
Defendemos o que Damico (2007) denominou de perspectiva crítica ligada à área da Educação Física, a qual relaciona a atividade física e a saúde com questões sociais mais amplas que impedem que os sujeitos tomem decisões mais saudáveis, tais como os condicionantes econômicos, culturais, étnicos e políticos.

Assim, concomitante ao fomento da prática regular de atividade física, deve haver a análise do contexto de vida dos sujeitos e o subsequente enfrentamento dos determinantes de sua saúde. Contrapõe, assim, a forma prescritivo-normativa com que a atividade física vem sendo 'receitada' aos sujeitos e que este documento reforça, pois concordamos com Carvalho (2005) ao afirmar que somente através de transformações econômicas, sociais e políticas, resultando em padrões saudáveis de existência, é que se produz saúde.

\section{Referências}

ANDRADE, C. L.; WIIK, C. B.; VASCONCELLOS, M.

P. C. Corpo e cultura e corpo e saúde: análise do suplemento semana de um jornal paulista. Saúde e Sociedade, São Paulo, v. 13, n. 3, P. 58-67, set-dez. 2004.

BAGRICHEVSKY, M.; ESTEVÃO, A. Os sentidos da saúde e a educação física: apontamentos preliminares. Arquivos em Movimento, Rio de janeiro, v. 1, n. 1, p. 65-74, jan./jun. 2005.

BARROS, J. A. C. Pensando o processo saúde doença: a que responde o modelo biomédico? Saúde e Sociedade, São Paulo, v. 11, n. 1, p. 1-11, 2002.

BRASIL. Ministério da Saúde. Secretaria de Vigilância em Saúde. Política nacional de promoção da saúde.Brasília, 2006.

BUSS, P. M. Promoção da saúde e qualidade de vida. Ciência \& Saúde Coletiva, Rio de Janeiro, v. 5, n. 1, p. 163-77, 2000.

BUSS, P. M. Promoção da saúde na infância e adolescência. Revista Brasileira de Saúde Materno Infantil, Recife, v. 1, n. 3, p. 279-82, set.-dez., 2001.

BUSS, P. M. Uma introdução ao conceito de promoção de saúde. In: CZERESNIA, D.; FREITAS, C. M. (Orgs.). Promoção da saúde: conceitos, reflexões, tendências. Rio de Janeiro: Fiocruz, 2003. 
BUSS, P. M. Apresentação em aula no curso de especialização em Promoção da Saúde e Desenvolvimento Social da Escola Nacional de Saúde Pública Sergio Arouca da Fundação Oswaldo Cruz, 2007.

BUSS, P. M.; PELLEGRINI FILHO, A. A saúde e seus determinantes sociais. Physis, Rio de Janeiro, v. 17, n. 1, p. 77-93, 2007.

CAMPOS, G. W.; BARROS, R. B.; CASTRO, A. M. Avaliação de política nacional de promoção da saúde. Ciência e Saúde Coletiva, Rio de Janeiro, v. 9, n. 3, p. 745-9, 2004.

CARVALHO, A. I. Princípios e prática da promoção da saúde no Brasil. Caderno de Saúde Pública, Rio de Janeiro, v. 24, n. 1, p. 4-5, jan. 2008.

CARVALHO, Y. M. Educação física e saúde coletiva: uma introdução. In: LUZ, M. T. Novos saberes e práticas em saúde coletiva: estudos sobre racionalidade médicas e atividades corporais. 2 ed. São Paulo: Hucitec, 2005, p. 19-34.

CASTIEL, L. D. Quem vive mais, morre menos? estilo de risco e promoção de saúde. In: BAGRICHEVSKY, M.; PALMA, A.; ESTEVÃO, A. (orgs.). A saúde em debate na educação física. Blumenau: Edibes, 2003. p. 79-97.

CONSELHO FEDERAL DE EDUCAÇÃO FÍSICA. Carta brasileira de prevenção integrada na área da saúde na perspectiva da educação física. 2 ed. Rio de Janeiro, 2006.

CZERESNIA, D. O conceito de saúde e a diferença entre prevenção e promoção. In: CZERESNIA, D., FREITAS C. M. (orgs.). Promoção da saúde: conceitos, reflexões, tendências. Rio de Janeiro: Fiocruz, 2003.

DAMICO, J. G. S. Das possibilidades às incertezas: instrumentos para intervenção do profissional de educação física no posto de saúde. In: FRAGA, A. B.; WACHS, F. Educação física e saúde coletiva: políticas de formação e perspectivas de intervenção. 2.ed. Porto Alegre: Editora da UFRGS, 2007, p. 73-86.

DARIDO, S. C.; SANCHES NETO, L. O contexto da educação física na escola. In: DARIDO, S. C.; RANGEL, I. C. A. (coords.). Educação física na escola: implicações para a prática pedagógica. Rio de Janeiro: Guanabara Koogan, 2005, p. 1-24.
DESLANDES, S. F. Prefácio. In: GONÇALVES, A. et al. Conhecendo e discutindo saúde coletiva e atividade física. Rio de Janeiro: Guanabara Koogan, 2004, p. 1.

FARINATTI, P. T. V.; FERREIRA, M. S. Saúde, promoção da saúde e educação física: conceitos, princípios e aplicações. Rio de Janeiro: Ed. UERJ, 2006.

FERREIRA, M. S.; NAJAR, A. L. Programas e campanhas de promoção da atividade física. Ciência \& Saúde Coletiva, Rio de Janeiro, v. 10, supl., 2005, p. 207-19.

FERREIRA, J. R.; BUSS, P. M. O que o desenvolvimento local tem a ver com a promoção da saúde? In: ZANCAN, L.; BODSTEIN, R.; MARCONDES, W. B. (orgs.). Promoção da saúde como caminho para o desenvolvimento local: a experiência em manguinhos-RJ. Rio de Janeiro: Abrasco/Fiocruz, p. 115-37, 2002.

MARCONDES, W. B. A convergência de referências na promoção da saúde. Saúde e Sociedade, São Paulo, v. 13, n. 1, p.5-13, jan.-abr. 2004.

ORGANIZAÇÃO MUNDIAL DA SAÚDE. Carta de Ottawa, 1986. Disponível em: <http://www.opas.org. br/coletiva/carta.cfm>. Acesso em: 18 dez. 2007.

PALMA, A.; BAGRICHEVSKY, M.; ESTEVÃO, A. Considerações teóricas acerca das questões relacionadas à promoção de saúde. In:

BAGRICHEVSKY, M.; PALMA, A.; ESTEVÃO, A. (orgs.). A saúde em debate na educação física. Blumenau: Edibes, 2003, p. 15-31.

PALMA, A. et al. Comportamentos de risco e vulnerabilidade entre estudantes de educação física. Revista Brasileira de Epidemiologia, São Paulo, v. 10, n. 1, p. 117-26, mar. 2007.

PEDROSA, J. I. S. Perspectivas na avaliação em promoção da saúde: uma abordagem institucional. Ciência e Saúde Coletiva, Rio de Janeiro, v. 9, n. 3, 2004, p. 617-26.

PEDROSA, J. I. S. Promoção da saúde e educação em saúde. In: CASTRO, A.; MALO, M. (orgs.) SUS: ressignificando a promoção da saúde. São Paulo: Hucitec/OPAS, p. 77-95, 2006. 
PERES, F. F. et al. Lazer, esporte e cultura na agenda local: a experiência de promoção da saúde em Manguinhos. Ciência \& Saúde Coletiva, Rio de Janeiro, v. 10, n. 3, p. 757-69, 2005.

REIS, I. N. C. Promoção da saúde e reorientação de serviço: a experiência do Centro de Saúde Escola Germano Sinval de Faria. In: ZANCAN, L.; BODSTEIN, R.; MARCONDES, W. B. (orgs.). Promoção da saúde como caminho para o desenvolvimento local: a experiência em Manguinhos-RJ. Rio de Janeiro: Abrasco/Fiocruz, 2002, p. 111-157.

ROSEN, G. Uma história da saúde pública. São Paulo: Hucitec, 1994.

STOTZ, E. N.; ARAÚJO, J. W. G. Promoção da saúde e cultura política: a reconstrução do consenso. Saúde e Sociedade, São Paulo, v. 13, n. 2, p. 5-19, maio-ago.

2004.

TEMPORÃO, J. G. Discurso de posse no cargo de ministro da saúde. Disponível em: http://www. fiocruz.br/ccs/cgi/cgilua.exe/sys/start.htm?infoid=76 9\&sid=9\&tpl=printerview. Acesso em 24 Jun. 2009.

ZIONI, F.; WESTPHAL, M. F. O enfoque dos determinantes sociais de saúde sob o ponto de vista da teoria social. Saúde e Sociedade, São Paulo, v. 16, n. 3, p. 26-34, 2007.

Recebido em: 30/06/2008

Reapresentado em: 03/01/2009

Aprovado em: 15/01/2009 